Journal of Science

\title{
Cubic Transmuted Power Function Distribution
}

\author{
Saiful Islam ANSARI ${ }^{1}$, ${ }^{(0)}$ Monjed Hisham SAMUH ${ }^{2, *(D)}$, Abouzar BAZYARI $^{3}$ \\ ${ }^{1}$ University of Tabuk, Department of Statistics, Tabuk, Saudi Arabia \\ ${ }^{2}$ Palestine Polytechnic University, Department of Applied Mathematics \& Physics, Hebron, Palestine \\ ${ }^{3}$ Persian Gulf University, Department of Statistics, Bushehr, Iran
}
Highlights
- The paper focuses on generating a new family of distribution.
- The objective of the proposed distribution is to better model real lifetime data.
- The validity of the distribution is tested by some real data that are derived from the literature.
- The proposed distribution outperforms other similar distributions used to model lifetime data.

\begin{tabular}{l} 
Article Info \\
\hline \\
Received: $15 / 10 / 2018$ \\
Accepted: $02 / 05 / 2019$ \\
Keywords \\
Power density function \\
Cubic transmuted distribution \\
Order statistic \\
Reliability function \\
Moment generating function
\end{tabular}

\section{INTRODUCTION}

Fitting distributions to data in real world applications is of major concern in the science of modelling. Many statisticians in the literature strive to generalize some existing distributions by introducing new parameters to the base distributions. The idea behind this is to make these distributions more flexible and more practicable, and thus to find the true nature of the data and how it behaves.

A lot of distributions have been suggested over the years to analyze and model real data. Skew-normal distribution [1] is developed by introducing the skewness parameter to the base normal distribution. Some researchers (see for example [2-6]) investigated the generalized skew-normal distribution.

Recently, transmuted family of distributions are investigated. Using the quadratic rank transmutation map suggested by [7,8] developed the transmuted extreme value distribution, [9] introduced the transmuted generalized inverted exponential distribution, [10] developed the transmuted Rayleigh distribution, [11] studied the transmuted inverse exponential distribution, and [12] generalized the transmuted power function distribution (See also [13]). Maximum likelihood estimation and some statistical properties (moments, order statistics, reliability and hazard functions, etc.) of these distributions are discussed therein. More recently, a new family of transmutation map, named cubic rank transmutation, is introduced by [14]. They developed 
the cubic rank transmuted log-logistic and cubic rank transmuted Weibull distributions. [15] derived the cubic transmuted Weibull distribution and discussed some of its statistical relations. [16] studied the cubic transmuted exponential distribution. In this paper, as the power function distribution is a well-known distribution in reliability analysis particularly in electrical component data [17], and to expand its uses for modelling various types of data, cubic transmuted power function distribution is developed.

Definition 1. The random variable (r.v) X follows a power function distribution, with parameters $\beta$ and $\alpha$, if its probability density function ( $p d f)$ is defined as

$$
g(x ; \alpha, \beta)=\frac{\alpha}{\beta^{\alpha}} x^{\alpha-1} ; \quad x<\beta, \quad \alpha, \beta>0 .
$$

The corresponding cumulative distribution function (cdf) of the power function distribution is

$$
G(x ; \alpha, \beta)=\left(\frac{x}{\beta}\right)^{\alpha} ; \quad x<\beta, \quad \alpha, \beta>0 .
$$

For the distribution statistical properties and some of its applications one may refer to [17-19].

Definition 2. A transmuted r.v $X$ has the $c d f$

$$
F(x)=(1+\lambda) G(x)-\lambda G^{2}(x), \quad|\lambda| \leq 1,
$$

where $G(x)$ is the base cdf of $X$. At $\lambda=0, G(x)$, the base distribution, is obtained.

According to Definitions 1 and 2, transmuted power function distribution can be defined. For a detailed study of the distribution see $[12,13]$.

The rest of this manuscript is formed as follows. The cdf and pdf of the cubic transmuted power function distribution are derived in Section 2. Some statistical properties including moments and some descriptive measures are investigated in Section 3. In Section 4 the order statistics are discussed. The maximum likelihood estimation and moment matching technique are described in Section 5. In Section 6, the properties of the estimators are investigated via Monte-Carlo simulation. At last, the superiority of the new distribution as compared to other existing models is proved empirically by means of three data sets in Section 7 .

\section{CUBIC TRANSMUTED POWER FUNCTION DISTRIBUTION}

Definition 3. A cubic transmuted $r . v X$ has the cdf

$$
F(x)=(1+\lambda) G(x)-2 \lambda G^{2}(x)+\lambda G^{3}(x), \quad|\lambda| \leq 1,
$$

where $G(x)$ is the base cdf of $X$. At $\lambda=0, G(x)$, the base distribution, is obtained.

Definition 4. The cdf of the cubic transmuted power function distribution (CTPFD) is

Accordingly, the $p d f$ is

$$
F(x ; \alpha, \beta, \lambda)=\frac{1+\lambda}{\beta^{\alpha}} x^{\alpha}-\frac{2 \lambda}{\beta^{2 \alpha}} x^{2 \alpha}+\frac{\lambda}{\beta^{3 \alpha}} x^{3 \alpha} ; \quad 0<x<\beta, \quad \alpha>0, \quad|\lambda| \leq 1 .
$$

$$
f(x ; \alpha, \beta, \lambda)=\frac{\alpha(\lambda+1)}{\beta^{\alpha}} x^{\alpha-1}-\frac{4 \alpha \lambda}{\beta^{2 \alpha}} x^{2 \alpha-1}+\frac{3 \alpha \lambda}{\beta^{3 \alpha}} x^{3 \alpha-1} .
$$

The pdf curves and their corresponding cdf curves of the CTPFD for distinct values of $\lambda, \beta$, and $\alpha$ are shown in Figure 1. It is evident that the new distribution is much more flexible than the power function and transmuted power function distributions.

Definition 5. The reliability function (survival function) measures the probability that a production will outrun for a given time duration. Mathematically, it is the complement of the cdf; that is,

$$
R(x)=1-F(x) \text {. }
$$



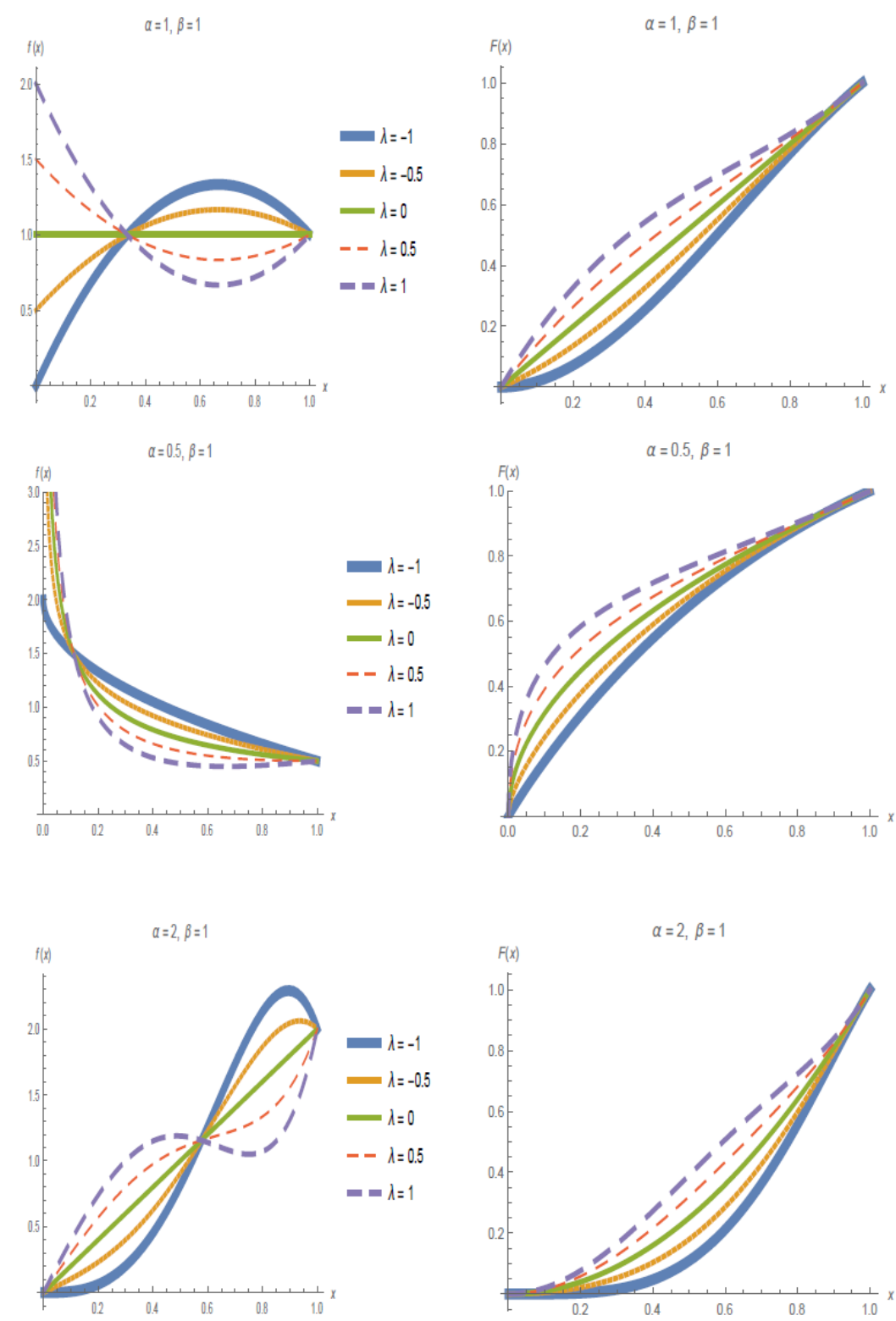

Figure 1. The pdf's curves (left panel) and their corresponding cdf curves (right panel) of the CTPFD for distinct values of $\alpha, \beta$, and $\lambda$

The reliability function is the most common used function in life data analysis. For the CTPFD, the reliability function is

$$
R(x)=1-\frac{1+\lambda}{\beta^{\alpha}} x^{\alpha}+\frac{2 \lambda}{\beta^{2 \alpha}} x^{2 \alpha}-\frac{\lambda}{\beta^{3 \alpha}} x^{3 \alpha} .
$$

Definition 6. The hazard function (or referred as failure rate) is the ratio of $f(x)$ to the reliability function $R(x)$ :

For the CTPFD,

$$
h(x)=\frac{f(x)}{R(x)}
$$

$$
h(x)=\alpha x^{\alpha-1}\left(\frac{\left(\beta^{\alpha}-2 x^{\alpha}\right) \lambda}{\beta^{2 \alpha}+\lambda x^{2 \alpha}-\lambda \beta^{\alpha} x^{\alpha}}+\frac{1}{\beta^{\alpha}-x^{\alpha}}\right) .
$$

Plot of hazard rate function for selected parameter values is displayed in Figure 2. 

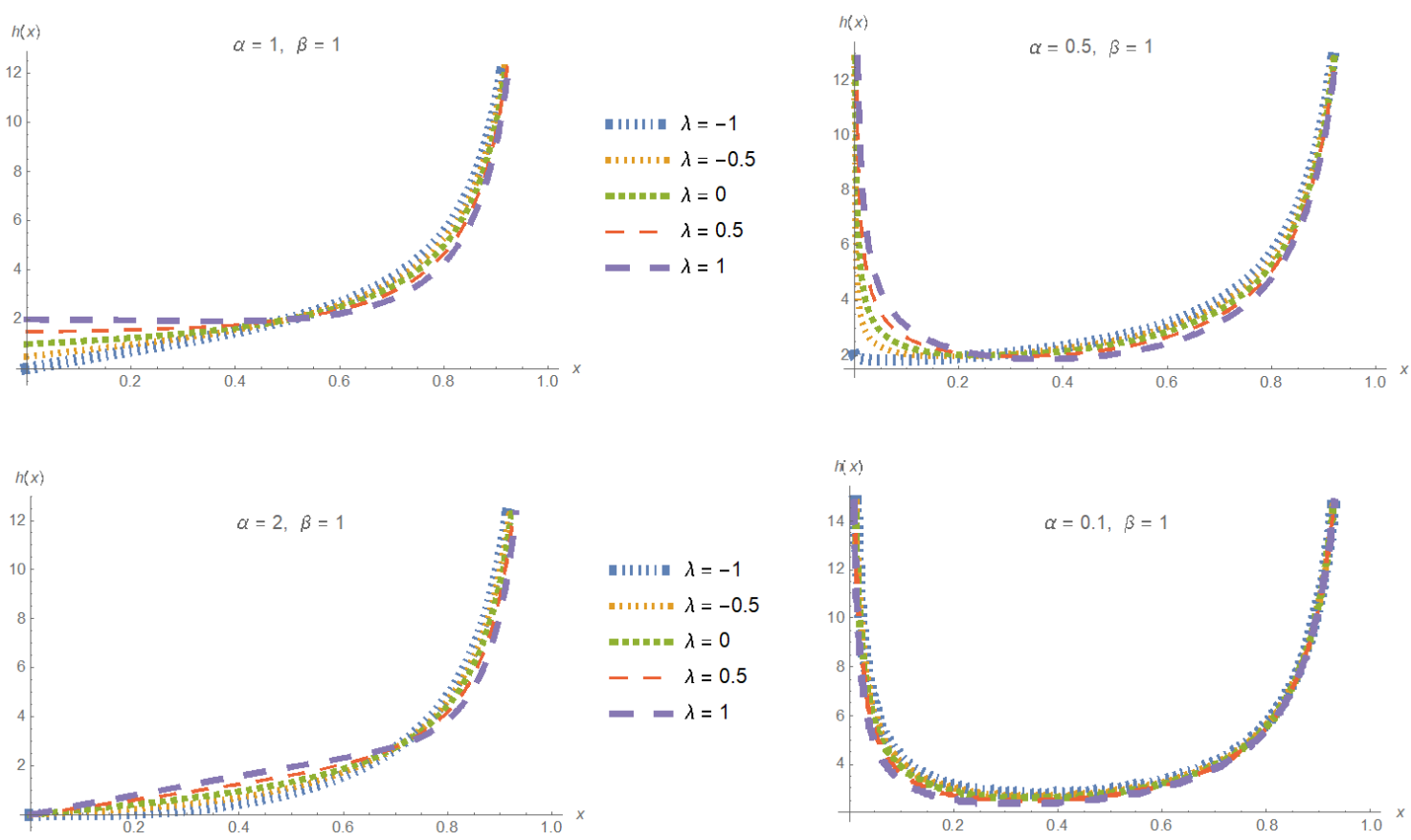

Figure 2. Plots of the CTPFD hazard rate for distinct values of $\alpha, \beta$, and $\lambda$

Definition 7. Given existence of a production until time $x$, the cumulative hazard function is the probability of failure at $x$. It is defined as

$$
H(x)=\int_{-\infty}^{x} h(t) d t
$$

It can be redefined as

$$
H(x)=-\ln (1-F(x))
$$

For the CTPFD,

$$
H(x)=-\ln \left(1-\frac{1+\lambda}{\beta^{\alpha}} x^{\alpha}+\frac{2 \lambda}{\beta^{2 \alpha}} x^{2 \alpha}-\frac{\lambda}{\beta^{3 \alpha}} x^{3 \alpha}\right)
$$

Definition 8. Let $T$ be a (continuous) r.v denotes the life-time of a production and presume that this production went wrong sometime before $t$. Then the time elapsed since the failure of $T$ knowing that the production went wrong sometime before $t$ is measured by the r.v $t-T \mid T \leq t$. The expectation of this $r . v$ is called the mean past lifetime (MPL) of T. MPL is defined as

$$
k(t)=E(t-T \mid T \leq t)=\frac{\int_{0}^{t} F(x) d x}{F(t)} .
$$

For the CTPFD, the MPL is given by

$$
k(t)=t\left(\frac{\frac{\lambda+1}{\alpha+1}+\frac{\lambda t^{2 \alpha}}{(3 \alpha+1) \beta^{2 \alpha}}-\frac{2 \lambda t^{\alpha}}{(2 \alpha+1) \beta^{\alpha}}}{(\lambda+1)+\frac{\lambda t^{2 \alpha}}{\beta^{2 \alpha}}-\frac{2 \lambda t^{\alpha}}{\beta^{\alpha}}}\right) .
$$

\section{SOME STATISTICAL RELATIONS OF THE DISTRIBUTION}

Some important statistical relations of the CTPFD are obtained in this section. This include moments, moment generating function, and some descriptive measures. 


\subsection{Moments}

Definition 9. Let $X$ a r.v. The $r^{\text {th }}$ moment about the origin is defined as

$$
E\left(X^{r}\right)=\int_{-\infty}^{\infty} x^{r} f(x) d x .
$$

Lemma 1. If $X$ is distributed as CTPFD, then

$$
E\left(X^{r}\right)=\frac{\alpha \beta^{r}\left(6 \alpha^{2}+r(5-2 \lambda) \alpha+r^{2}\right)}{(\alpha+r)(2 \alpha+r)(3 \alpha+r)}
$$

Proof. The proof is straightforward.

Giving $r=1$ in Lemma 1, the mean of $X$ is obtained.

$$
\mu=E(X)=\frac{\alpha \beta\left(6 \alpha^{2}+(5-2 \lambda) \alpha+1\right)}{(\alpha+1)(2 \alpha+1)(3 \alpha+1)} .
$$

The variance may acquire using the relation $\operatorname{Var}(X)=E\left(X^{2}\right)-\mu^{2}$. Thus,

$$
\operatorname{Var}(X)=\frac{\alpha \beta^{2}\left(6 \alpha^{2}+2(5-2 \lambda) \alpha+4\right)}{(\alpha+2)(2 \alpha+2)(3 \alpha+2)}-\left(\frac{\alpha \beta\left(6 \alpha^{2}+(5-2 \lambda) \alpha+1\right)}{(\alpha+1)(2 \alpha+1)(3 \alpha+1)}\right)^{2} .
$$

\subsection{Conditional Moments}

The $r^{\text {th }}$ conditional moment of CTPFD is defined as

where

$$
E\left(X^{r} \mid X>t\right)=\frac{1}{1-F(t)} \int_{t}^{\infty} x^{r} f(x) d x,
$$

$$
\begin{aligned}
\int_{t}^{\infty} x^{r} f(x) d x & =\int_{t}^{\infty} x^{r} \frac{\alpha(\lambda+1)}{\beta^{\alpha}} x^{\alpha-1}-\frac{4 \alpha \lambda}{\beta^{2 \alpha}} x^{2 \alpha-1}+\frac{3 \alpha \lambda}{\beta^{3 \alpha}} x^{3 \alpha-1} d x \\
& =\frac{\alpha(\lambda+1)}{\beta^{\alpha}} \int_{t}^{\infty} x^{r+\alpha-1} d x-\frac{4 \alpha \lambda}{\beta^{2 \alpha}} \int_{t}^{\infty} x^{r+2 \alpha-1} d x+\frac{3 \alpha \lambda}{\beta^{3 \alpha}} \int_{t}^{\infty} x^{r+3 \alpha-1} d x \\
& =\frac{\alpha(\lambda+1)}{\beta^{\alpha}}\left(\frac{-t^{r+\alpha}}{r+\alpha}\right)-\frac{4 \alpha \lambda}{\beta^{2 \alpha}}\left(\frac{-t^{r+2 \alpha}}{r+2 \alpha}\right)+\frac{3 \alpha \lambda}{\beta^{3 \alpha}}\left(\frac{-t^{r+3 \alpha}}{r+3 \alpha}\right),
\end{aligned}
$$

provided that $r<\alpha$.

Therefore,

$$
E\left(X^{r} \mid X>t\right)=\frac{-\frac{\alpha(\lambda+1)}{\beta^{\alpha}}\left(\frac{t^{r+\alpha}}{r+\alpha}\right)+\frac{4 \alpha \lambda}{\beta^{2 \alpha}}\left(\frac{t^{r+2 \alpha}}{r+2 \alpha}\right)-\frac{3 \alpha \lambda}{\beta^{3 \alpha}}\left(\frac{t^{r+3 \alpha}}{r+3 \alpha}\right)}{1-\left(\frac{1+\lambda}{\beta^{\alpha}} t^{\alpha}-\frac{2 \lambda}{\beta^{2 \alpha}} t^{2 \alpha}+\frac{\lambda}{\beta^{3 \alpha}} t^{3 \alpha}\right)} .
$$

In particular,

$$
E(X \mid X>t)=\frac{-\frac{\alpha(\lambda+1)}{\beta^{\alpha}}\left(\frac{t^{1+\alpha}}{1+\alpha}\right)+\frac{4 \alpha \lambda}{\beta^{2 \alpha}}\left(\frac{t^{1+2 \alpha}}{1+2 \alpha}\right)-\frac{3 \alpha \lambda}{\beta^{3 \alpha}}\left(\frac{t^{1+3 \alpha}}{1+3 \alpha}\right)}{1-\left(\frac{1+\lambda}{\beta^{\alpha}} t^{\alpha}-\frac{2 \lambda}{\beta^{2 \alpha}} t^{2 \alpha}+\frac{\lambda}{\beta^{3 \alpha}} t^{3 \alpha}\right)}
$$


The so-called mean residual lifetime of the CTPFD is obtained by $E(X \mid X>t)-t$.

\subsection{Moment Generating Function (mgf)}

Definition 10. The mgf of a r.v $X$ is evaluated as

$$
M(t)=E\left(e^{t X}\right)=\int_{-\infty}^{\infty} e^{t x} f(x) d x .
$$

Lemma 2. If $X$ is distributed as CTPFD, then the mgf of $X$ is

$$
M(t)=\alpha \sum_{n=0}^{\infty} \frac{(t \beta)^{n}}{n !}\left(\frac{1+\lambda}{n+\alpha}-\frac{4 \lambda}{n+2 \alpha}+\frac{3 \lambda}{n+3 \alpha}\right) .
$$

Proof. Applying Definition 10, the mgf of $X$ is derived as

$$
\begin{aligned}
M(t) & =\int_{0}^{\beta} e^{t x}\left(\frac{\alpha(\lambda+1)}{\beta^{\alpha}} x^{\alpha-1}-\frac{4 \alpha \lambda}{\beta^{2 \alpha}} x^{2 \alpha-1}+\frac{3 \alpha \lambda}{\beta^{3 \alpha}} x^{3 \alpha-1}\right) d x \\
& =\frac{\alpha(\lambda+1)}{\beta^{\alpha}} \int_{0}^{\beta} e^{t x} x^{\alpha-1} d x-\frac{4 \alpha \lambda}{\beta^{2 \alpha}} \int_{0}^{\beta} e^{t x} x^{2 \alpha-1} d x+\frac{3 \alpha \lambda}{\beta^{3 \alpha}} \int_{0}^{\beta} e^{t x} x^{3 \alpha-1} d x \\
& =\frac{\alpha(\lambda+1)}{\beta^{\alpha}} I_{1}-\frac{4 \alpha \lambda}{\beta^{2 \alpha}} I_{2}+\frac{3 \alpha \lambda}{\beta^{3 \alpha}} I_{3},
\end{aligned}
$$

where

$$
\begin{aligned}
I_{1} & =\int_{0}^{\beta} e^{t x} x^{\alpha-1} d x \\
& =\int_{0}^{\beta} x^{\alpha-1}\left(\sum_{n=0}^{\infty} \frac{(t x)^{n}}{n !}\right) d x=\sum_{n=0}^{\infty} \frac{t^{n}}{n !} \int_{0}^{\beta} x^{n+\alpha-1} d x \\
& =\sum_{n=0}^{\infty} \frac{t^{n}}{n !}\left[\frac{x^{n+\alpha}}{n+\alpha}\right]_{0}^{\beta}=\sum_{n=0}^{\infty} \frac{t^{n} \beta^{n+\alpha}}{n !(n+\alpha)} .
\end{aligned}
$$

Similarly,

$$
I_{2}=\sum_{n=0}^{\infty} \frac{t^{n} \beta^{n+2 \alpha}}{n !(n+2 \alpha)} \quad \text { and } \quad I_{3}=\sum_{n=0}^{\infty} \frac{t^{n} \beta^{n+3 \alpha}}{n !(n+3 \alpha)}
$$

Hence the proof is completed.

\subsection{Geometric Mean}

Definition 11. The geometric mean $G$ of a r.v X can be obtained such that

$$
\log G=\int_{-\infty}^{\infty} \log (x) f(x) d x .
$$

Lemma 3. If $X$ is distributed as CTPFD, then the geometric mean of $X$ is given by

Proof.

$$
G=\text { Antilog }\left[\frac{1}{6}(3 \alpha \log \beta-(3+\lambda))\right] .
$$




$$
\begin{aligned}
\log G & =\int_{0}^{\beta} \log (x)\left(\frac{\alpha(\lambda+1)}{\beta^{\alpha}} x^{\alpha-1}-\frac{4 \alpha \lambda}{\beta^{2 \alpha}} x^{2 \alpha-1}+\frac{3 \alpha \lambda}{\beta^{3 \alpha}} x^{3 \alpha-1}\right) d x \\
& =\frac{\alpha}{\beta^{3 \alpha}}\left((\lambda+1) \beta^{2 \alpha} \int_{0}^{\beta} \log (x) x^{\alpha-1} d x-4 \lambda \beta^{\alpha} \int_{0}^{\beta} \log (x) x^{2 \alpha-1} d x+3 \lambda \int_{0}^{\beta} \log (x) x^{3 \alpha-1} d x\right) \\
& =\frac{\alpha}{\beta^{3 \alpha}}\left((\lambda+1) \beta^{2 \alpha} J_{1}-4 \lambda \beta^{\alpha} J_{2}+3 \lambda J_{3}\right) .
\end{aligned}
$$

For $i=1,2,3$, it can be shown, using the formula for integration by parts, that

Therefore, after simple algebra,

$$
J_{i}=\frac{\beta^{i \alpha}}{(i \alpha)^{2}}(i \alpha \log \beta-1) .
$$

$$
\log G=\frac{1}{6}(3 \alpha \log \beta-(3+\lambda)) .
$$

Thus, the geometric mean of $X$ is given by

$$
G=\text { Antilog }\left[\frac{1}{6}(3 \alpha \log \beta-(3+\lambda))\right] .
$$

\subsection{Harmonic Mean}

Definition 12. The harmonic mean $H$ of a r.v $X$ can be obtained such that

$$
\frac{1}{H}=\int_{-\infty}^{\infty} \frac{1}{x} f(x) d x
$$

Lemma 4. If $X$ is distributed as CTPFD, then the harmonic mean of $X$ is given by

$$
H=\frac{\beta(\alpha-1)(2 \alpha-1)(3 \alpha-1)}{\alpha\left(6 \alpha^{2}-(5-2 \alpha) \lambda+1\right)} .
$$

Proof. The proof is straightforward.

\subsection{Median and Mode}

Definition 13. The median $M$ of a r.v $X$ can be defined such that

$$
\int_{-\infty}^{M} f(x) d x=\int_{M}^{\infty} f(x) d x=\frac{1}{2} .
$$

Lemma 5. If $X$ is distributed as CTPFD, then the median of $X$ can be located by solving the equation

$$
\lambda M^{3 \alpha}-2 \lambda \beta^{\alpha} M^{2 \alpha}+(1+\lambda) \beta^{2 \alpha} M^{\alpha}=\frac{\beta^{3 \alpha}}{2} .
$$

Definition 14. The mode of $X$ is the value of $x_{*}$ for which its probability distribution, $f(x)$, takes a largest value.

Lemma 6. If $X$ is distributed as CTPFD, then the mode of $X$ is given by

$$
x_{*}=\beta\left(\frac{\lambda(2 \alpha-1) \pm \sqrt{\lambda\left(16 \lambda \alpha^{2}-(25 \alpha-7) \lambda-3(3 \alpha-1)\right)}}{3 \lambda(3 \alpha-1)}\right)^{\frac{1}{\alpha}} \text {. }
$$


Proof. To find $x_{*}$ of CTPFD for which its pdf attains its maximum, take the 1st derivative of $f(x)$ and equating it to 0 . Then the value of $x, x_{*}$, that make $f^{\prime}\left(x_{*}\right)=0$ is the mode given that $f^{\prime \prime}\left(x_{*}\right)<0$. The first derivative is

$$
f^{\prime}(x)=\frac{\alpha(\lambda+1)(\alpha-1)}{\beta^{\alpha}} x^{\alpha-2}-\frac{4 \alpha \lambda(2 \alpha-1)}{\beta^{2 \alpha}} x^{2 \alpha-2}+\frac{3 \alpha \lambda(3 \alpha-1)}{\beta^{3 \alpha}} x^{3 \alpha-2} .
$$

At $x=x_{*}$, equate $f^{\prime}\left(x_{*}\right)=0$, and after simple algebra we get

$$
3 \lambda x_{*}^{2 \alpha}(3 \alpha-1)-4 \lambda \beta^{\alpha} x_{*}^{\alpha}(2 \alpha-1)+\beta^{2 \alpha}(\lambda+1)(\alpha-1)=0 .
$$

Let $y=x_{*}^{\alpha}$, we get

$$
3(3 \alpha-1) \lambda y^{2}-4(2 \alpha-1) \lambda \beta^{\alpha} y+(\alpha-1)(\lambda+1) \beta^{2 \alpha}=0,
$$

which is an equation in the quadratic form. Using the quadratic formula, we get

$$
y=\frac{2(2 \alpha-1) \lambda \beta^{\alpha} \pm \sqrt{\lambda \beta^{2 \alpha}\left(16 \lambda \alpha^{2}-(25 \alpha-7) \lambda-3(3 \alpha-1)\right)}}{3(3 \alpha-1) \lambda} .
$$

Thus,

$$
x_{*}=\beta\left(\frac{\lambda(2 \alpha-1) \pm \sqrt{\lambda\left(16 \lambda \alpha^{2}-(25 \alpha-7) \lambda-3(3 \alpha-1)\right)}}{3 \lambda(3 \alpha-1)}\right)^{\frac{1}{\alpha}} .
$$

If $f^{\prime \prime}\left(x_{*}\right)<0$, then $x_{*}$ is the mode.

\section{ORDER STATISTICS}

Definition 15. Let $F(x)$ be the cdf of a random sample $X_{1}, X_{2}, \ldots, X_{n}$ and $f(x)$ be its pdf. Reorder $X_{1}, X_{2}, \ldots, X_{n}$ such that $X_{(n)}>X_{(n-1)}>\cdots>X_{(1)}$, then $X_{(j)}$ is called the $j^{\text {th }}$ order statistic (o.s). Then, $X_{(j)}$ has the following marginal pdf:

$$
f_{X_{(j)}}(x)=n\left(\begin{array}{c}
n-1 \\
j-1
\end{array}\right) f(x)(F(x))^{j-1}(1-F(x))^{n-j} .
$$

The $1^{\text {st }}$ o.s, $X_{(1)}$, has the following marginal pdf

$$
f_{X_{(1)}}(x)=n f(x)(1-F(x))^{n-1} .
$$

The $n^{\text {th }}$ o.s, $X_{(n)}$, has the following marginal $p d f$

$$
f_{X_{(n)}}(x)=n f(x)(F(x))^{n-1} .
$$

Lemma 7. Suppose $X_{1}, X_{2}, \ldots, X_{n}$ be a r.s from CTPFD, then the $j^{\text {th }}$ o.s pdf is

$$
\begin{aligned}
f_{X_{(j)}}(x) & =n\left(\begin{array}{c}
n-1 \\
j-1
\end{array}\right) \alpha \beta^{-3 \alpha} x^{\alpha-1}\left((\lambda+1) \beta^{2 \alpha}+3 \lambda x^{2 \alpha}-4 \lambda \beta^{\alpha} x^{\alpha}\right) \\
& \left(\beta^{-3 \alpha} x^{\alpha}\left(\beta^{2 \alpha}(\lambda+1)+\left(x^{2 \alpha}-2 \beta^{\alpha} x^{\alpha}\right) \lambda\right)\right)^{j-1} \\
& \left(\beta^{-3 \alpha}\left(\beta^{\alpha}-x^{\alpha}\right)\left(\beta^{2 \alpha}+\left(x^{2 \alpha}-\beta^{\alpha} x^{\alpha}\right) \lambda\right)\right)^{n-j},
\end{aligned}
$$

The $1^{\text {st }}$ o.s pdf is 


$$
\begin{array}{r}
f_{X_{(1)}}(x)=\alpha n \beta^{-3 \alpha} x^{\alpha-1}\left((\lambda+1) \beta^{2 \alpha}+3 \lambda x^{2 \alpha}-4 \lambda \beta^{\alpha} x^{\alpha}\right) \\
\left(\beta^{-3 \alpha}\left(\beta^{\alpha}-x^{\alpha}\right)\left(\beta^{2 \alpha}+\lambda\left(x^{2 \alpha}-\beta^{\alpha} x^{\alpha}\right)\right)\right)^{n-1},
\end{array}
$$

and the $n^{\text {th }}$ o.s pdf is

$$
\begin{array}{r}
f_{X_{(n)}}(x)=\alpha n \beta^{-3 \alpha} x^{\alpha-1}\left((\lambda+1) \beta^{2 \alpha}+3 \lambda x^{2 \alpha}-4 \lambda \beta^{\alpha} x^{\alpha}\right) \\
\left(\beta^{-3 \alpha} x^{\alpha}\left((\lambda+1) \beta^{2 \alpha}+\lambda\left(x^{2 \alpha}-2 \beta^{\alpha} x^{\alpha}\right)\right)\right)^{n-1} .
\end{array}
$$

\section{ESTIMATION AND INFERENCE}

\subsection{Maximum Likelihood Estimation (MLE)}

Let $x_{1}, x_{2}, \ldots, x_{n}$ be observations selected from CTPFD with parameters $\alpha, \beta$, and $\lambda$. Let $\Theta=(\alpha, \beta, \lambda)$ be the $3 \times 1$ parameter vector. The likelihood function of CTPFD for the parameter vector $\Theta$ can be written as

$$
\begin{aligned}
L\left(\Theta ; x_{1}, x_{2}, \ldots, x_{n}\right) & =\prod_{i=1}^{n} f\left(x_{i} ; \alpha, \beta, \lambda\right) \\
& =\prod_{i=1}^{n}\left(\frac{\alpha(\lambda+1)}{\beta^{\alpha}} x_{i}^{\alpha-1}-\frac{4 \alpha \lambda}{\beta^{2 \alpha}} x_{i}^{2 \alpha-1}+\frac{3 \alpha \lambda}{\beta^{3 \alpha}} x_{i}^{3 \alpha-1}\right) .
\end{aligned}
$$

The total log-likelihood function for $\Theta$ will be

$$
\begin{aligned}
l\left(\Theta ; x_{1}, \ldots, x_{n}\right) & =\ln L\left(\Theta ; x_{1}, \ldots, x_{n}\right) \\
= & \sum_{i=1}^{n} \ln \left(\alpha(\lambda+1) \beta^{-\alpha} x_{i}^{\alpha-1}-4 \alpha \lambda \beta^{-2 \alpha} x_{i}^{2 \alpha-1}+3 \alpha \lambda \beta^{-3 \alpha} x_{i}^{3 \alpha-1}\right) .
\end{aligned}
$$

Differentiating the $\log$ likelihood function with respect to $\alpha, \beta$, and $\lambda$ results in

$$
\begin{aligned}
\frac{\partial}{\partial \alpha} l\left(\Theta ; x_{1}, \ldots, x_{n}\right)= & \frac{n}{\alpha}-3 n \ln \beta+3 \sum_{i=1}^{n} \ln x_{i} \\
& +\sum_{i=1}^{n} \frac{2 \beta^{\alpha}\left(\ln \beta-\ln x_{i}\right)\left((\lambda+1) \beta^{\alpha}-2 \lambda x_{i}^{\alpha}\right)}{(\lambda+1) \beta^{2 \alpha}+\lambda x_{i}^{\alpha}\left(3 x_{i}^{\alpha}-4 \beta^{\alpha}\right)} \\
\frac{\partial}{\partial \beta} l\left(\Theta ; x_{1}, \ldots, x_{n}\right) & =\frac{\alpha}{\beta} \sum_{i=1}^{n} \frac{\lambda x_{i}^{\alpha}\left(8 \beta^{\alpha}-9 x_{i}^{\alpha}\right)-(\lambda+1) \beta^{2 \alpha}}{(\lambda+1) \beta^{2 \alpha}+\lambda x_{i}^{\alpha}\left(3 x_{i}^{\alpha}-4 \beta^{\alpha}\right)} \\
\frac{\partial}{\partial \lambda} l\left(\Theta ; x_{1}, \ldots, x_{n}\right)= & \sum_{i=1}^{n} \frac{\beta^{2 \alpha}-4 \beta^{\alpha} x_{i}^{\alpha}+3 x_{i}^{2 \alpha}}{\lambda \beta^{2 \alpha}+\beta^{2 \alpha}+4 \lambda \beta^{\alpha} x_{i}^{\alpha}+3 \lambda x_{i}^{2 \alpha}} .
\end{aligned}
$$

The MLEs of $\alpha, \beta$, and $\lambda$ can be found by equating these partial derivatives to zero. However, these equations cannot be solved analytically, and statistical software can be used to solve them numerically. Iterative techniques such as Newton-Raphson algorithm can be used to obtain the estimates.

\subsection{Moment Matching Estimation (MME)}

Let $x_{1}, x_{2}, \ldots, x_{n}$ be observations selected from CTPFD with parameters $\alpha, \beta$, and $\lambda$. The formula for the $r^{\text {th }}$ - moment is provided in Lemma 1 . The MMEs for the CTPFD may acquire by coinciding the $1^{\text {st }}$ three theoretical central moments with their corresponding (sample) moments. That is, the estimators $\hat{\alpha}, \hat{\beta}$, and $\hat{\lambda}$ may obtain by solving the following system of equations: 


$$
\begin{aligned}
& \frac{\alpha \beta\left(6 \alpha^{2}-2 \alpha \lambda+5 \alpha+1\right)}{(\alpha+1)(2 \alpha+1)(3 \alpha+1)}=\frac{1}{n} \sum_{i=1}^{n} x_{i}, \\
& \frac{\alpha \beta^{2}\left(3 \alpha^{2}-2 \alpha \lambda+5 \alpha+2\right)}{(\alpha+1)(\alpha+2)(3 \alpha+2)}=\frac{1}{n} \sum_{i=1}^{n} x_{i}^{2}, \\
& \frac{\alpha \beta^{3}\left(2 \alpha^{2}-2 \alpha \lambda+5 \alpha+3\right)}{(\alpha+1)(\alpha+3)(2 \alpha+3)}=\frac{1}{n} \sum_{i=1}^{n} x_{i}^{3} .
\end{aligned}
$$

However, these (non-linear) equations are not expressed in closed form. So, numerical methods should be used to find the solutions.

\subsection{Evaluating the Goodness of an Estimator}

Suppose $\hat{\theta}$ is an estimator used to estimate $\theta(\theta=\alpha, \beta$, or $\lambda)$, then the bias of $\hat{\theta}$ is given by

$$
\operatorname{Bias}(\hat{\theta})=E(\hat{\theta})-\theta
$$

and the mean square error of $\hat{\theta}$ is evaluated as

$$
\operatorname{MSE}(\hat{\theta})=E(\theta-\hat{\theta})^{2} .
$$

The performance of the estimator is evaluated via Monte-Carlo simulation.

Due to the unknown distribution of the derived estimator $\hat{\theta}$, empirical method is used to construct a confidence interval. An approximate $100(1-\gamma) \%$ of $\theta$ can be constructed as

$$
P\left(-Q_{\frac{\gamma}{2}} \leq \frac{\hat{\theta}-\theta}{S E(\theta)} \leq Q_{\frac{\gamma}{2}}\right)=1-\gamma,
$$

where $Q_{\gamma}$ is the $\gamma^{\text {th }}$ upper percentile of the empirical distribution of the corresponding estimator, and $\operatorname{SE}(\theta)=\sqrt{\operatorname{MSE}(\hat{\theta})}$ is the empirical standard error.

Therefore, the approximate $100(1-\gamma) \%$ confidence limits for $\alpha, \beta$ and $\lambda$ of the CTPFD are given, respectively, by

$$
\begin{aligned}
& P\left(\hat{\alpha}-Q_{\frac{\gamma}{2}} S E(\hat{\alpha}) \leq \alpha \leq \hat{\alpha}+Q_{\frac{\gamma}{2}} S E(\hat{\alpha})\right)=1-\gamma, \\
& P\left(\hat{\beta}-Q_{\frac{\gamma}{2}} S E(\hat{\beta}) \leq \beta \leq \hat{\beta}+Q_{\frac{\gamma}{2}} S E(\hat{\beta})\right)=1-\gamma, \\
& P\left(\hat{\lambda}-Q_{\frac{\gamma}{2}} S E(\hat{\lambda}) \leq \lambda \leq \hat{\lambda}+Q_{\frac{\gamma}{2}} S E(\hat{\lambda})\right)=1-\gamma .
\end{aligned}
$$

\section{SIMULATION STUDY}

To evaluate the properties of the MMEs and MLEs, a simulation study is carried out in this section. Sample of sizes 50, 150, and 300 from cubic transmuted power function distribution are considered. 2000 random samples are selected for each set up with the following combinations:

$$
(\alpha, \beta, \lambda)=\{(1,2,0.5),(2,1.5,0.5)\}
$$

This simulation study is carried out using the software $\mathrm{R}$ [20]. 
To generate random numbers from CTPFD, the following equation must be solved in terms of $x$ :

$$
\frac{x^{\alpha}}{\beta^{3 \alpha}}\left((\lambda+1) \beta^{2 \alpha}+\lambda x^{2 \alpha}-2 \lambda \beta^{\alpha} x^{\alpha}\right)=u,
$$

where $u$ is generated from a $U(0,1)$ standard uniform distribution.

Tables 1 and 2 present the averages of the estimates, the bias, the MSE, and 95\% confidence intervals (C.I) for the model parameters under the two methods of estimation; MME and MLE, respectively. It is obvious that, as $n$ increases, the MSE decreases. That is, the estimators are consistent. In addition, the width of the confidence interval gets narrower with increasing sample size.

Table 1. Mean of MMEs of all parameters, Bias, and MSE, for distinct values of $n$, and distinct setting of $(\alpha, \beta, \lambda)$

\begin{tabular}{|c|c|c|c|c|c|c|c|}
\hline \multirow{2}{*}{$\begin{array}{l}\text { Sample } \\
\text { size }\end{array}$} & \multirow[t]{2}{*}{$(\alpha, \beta, \lambda) \rightarrow$} & \multicolumn{3}{|c|}{$(1,2,0.5)$} & \multicolumn{3}{|c|}{$(2,1.5,0.5)$} \\
\hline & & $\alpha$ & $\beta$ & $\lambda$ & $\alpha$ & $\beta$ & $\lambda$ \\
\hline \multirow{5}{*}{50} & Estimate & 1.028 & 1.995 & 0.494 & 1.972 & 1.511 & 0.515 \\
\hline & Bias & 0.028 & -0.005 & -0.006 & -0.028 & 0.011 & 0.015 \\
\hline & MSE & 0.0330 & 0.0103 & 0.1755 & 0.1256 & 0.0014 & 0.2275 \\
\hline & C.I & $(0.67,1.38)$ & $(1.80,2.19)$ & $(-0.33,1.31)$ & $(1.28,2.67)$ & $(1.44,1.58)$ & $(-0.42,1.45)$ \\
\hline & Width & 0.71 & 0.40 & 1.64 & 1.39 & 0.15 & 1.87 \\
\hline \multirow{5}{*}{150} & Estimate & 1.012 & 2.001 & 0.506 & 1.973 & 1.511 & 0.513 \\
\hline & Bias & 0.012 & 0.001 & 0.006 & -0.027 & 0.011 & 0.013 \\
\hline & MSE & 0.0195 & 0.0038 & 0.1437 & 0.0725 & 0.0005 & 0.1578 \\
\hline & C.I & $(0.74,1.29)$ & $(1.88,2.12)$ & $(-0.24,1.25)$ & $(1.45,2.50)$ & $(1.471 .56)$ & $(-0.27,1.29)$ \\
\hline & Width & 0.55 & 0.24 & 1.49 & 1.06 & 0.09 & 1.56 \\
\hline \multirow{5}{*}{300} & Estimate & 1.001 & 2.004 & 0.486 & 1.988 & 1.508 & 0.527 \\
\hline & Bias & 0.001 & 0.004 & -0.014 & -0.012 & 0.008 & 0.027 \\
\hline & MSE & 0.0148 & 0.0021 & 0.1195 & 0.0461 & 0.0003 & 0.1098 \\
\hline & C.I & $(0.76,1.24)$ & $(1.91,2.09)$ & $(-0.19,1.16)$ & $(1.57,2.41)$ & $(1.48,1.54)$ & $(-0.12,1.18)$ \\
\hline & Width & 0.48 & 0.18 & 1.36 & 0.84 & 0.06 & 1.3 \\
\hline
\end{tabular}

Table 2. Mean of MLEs of all parameters, Bias, and MSE, for distinct values of $n$, and distinct setting of $(\alpha, \beta, \lambda)$

\begin{tabular}{|c|c|c|c|c|c|c|c|}
\hline \multirow{2}{*}{$\begin{array}{l}\text { Sample } \\
\text { size }\end{array}$} & \multirow[t]{2}{*}{$(\alpha, \beta, \lambda) \rightarrow$} & \multicolumn{3}{|c|}{$(1,2,0.5)$} & \multicolumn{3}{|c|}{$(2,1.5,0.5)$} \\
\hline & & $\alpha$ & $\beta$ & $\lambda$ & $\alpha$ & $\beta$ & $\lambda$ \\
\hline \multirow{5}{*}{50} & Estimate & 1.048 & 1.958 & 0.536 & 2.095 & 1.484 & 0.544 \\
\hline & Bias & 0.048 & -0.042 & 0.036 & 0.095 & -0.016 & 0.044 \\
\hline & MSE & 0.0404 & 0.0034 & 0.2544 & 0.1788 & 0.0005 & 0.2672 \\
\hline & C.I & $(0.64,1.44)$ & $(1.84,2.07)$ & $(-0.45,1.52)$ & $(1.27,2.92)$ & $(1.44,1.53)$ & $(-0.47,1.56)$ \\
\hline & Width & 0.79 & 0.23 & 1.98 & 1.66 & 0.09 & 2.03 \\
\hline \multirow{5}{*}{150} & Estimate & 1.016 & 1.987 & 0.518 & 2.024 & 1.495 & 0.507 \\
\hline & Bias & 0.016 & -0.013 & 0.018 & 0.024 & -0.005 & 0.007 \\
\hline & MSE & 0.0202 & 0.0003 & 0.1818 & 0.0840 & 0.0001 & 0.1825 \\
\hline & C.I & $(0.74,1.30)$ & $(1.95,2.02)$ & $(-0.32,1.35)$ & $(1.46,2.59)$ & $(1.48,1.51)$ & $(-0.33,1.34)$ \\
\hline & Width & 0.56 & 0.07 & 1.67 & 1.14 & 0.03 & 1.67 \\
\hline \multirow{5}{*}{300} & Estimate & 1.012 & 1.993 & 0.523 & 2.008 & 1.497 & 0.501 \\
\hline & Bias & 0.012 & -0.007 & 0.023 & 0.008 & -0.003 & 0.001 \\
\hline & MSE & 0.0112 & 0.0001 & 0.1047 & 0.0480 & 0.0000 & 0.1149 \\
\hline & C.I & $(0.80,1.22)$ & $(1.98,2.01)$ & $(-0.11,1.16)$ & $(1.58,2.44)$ & $(1.49,1.50)$ & $(-0.16,1.16)$ \\
\hline & Width & 0.41 & 0.04 & 1.27 & 0.86 & 0.01 & 1.33 \\
\hline
\end{tabular}

\section{DATA APPLICATION}

In this section, one artificial data set, and two real-life data sets are analyzed for the purpose of illustration to show the usefulness and flexibility of the CTPFD. These data sets are chosen because they really show in different fields that it is necessary to have nonnegative range. Here is the description of the data sets.

Data set I: This data set consists of 100 data points simulated from CTPFD with parameters $\alpha=1, \beta=2$, 
$\lambda=0.8$.

Data set II: This data corresponding to 72-hour acute salinity tolerance (LC50 values) of river marine invertebrates. It is taken from the R package "fitdistrplus" [21].

Data set III: It taken from [22]. It gives the failure times of 50 components (per 1000h). The observations are:

\begin{tabular}{lllllllllllll}
\hline 0.036 & 0.058 & 0.061 & 0.074 & 0.078 & 0.086 & 0.102 & 0.103 & 0.114 & 0.116 & 0.148 & 0.183 & 0.192 \\
0.254 & 0.262 & 0.379 & 0.381 & 0.538 & 0.570 & 0.574 & 0.590 & 0.618 & 0.645 & 0.961 & 1.228 & 1.600 \\
2.006 & 2.054 & 2.804 & 3.058 & 3.076 & 3.147 & 3.625 & 3.704 & 3.931 & 4.073 & 4.393 & 4.534 & 4.893 \\
6.274 & 6.816 & 7.896 & 7.904 & 8.022 & 9.337 & 10.94 & 11.02 & 13.88 & 14.73 & 15.08 & & \\
\hline
\end{tabular}

Some descriptive statistics for these data sets are provided in Table 3.

Table 3. Summary statistics for the three data sets

\begin{tabular}{c|cccccc}
\hline Data set & Min & $Q_{1}$ & Median & Mean & Q3 & Max \\
\hline Data set I & 0.003 & 0.232 & 0.733 & 0.805 & 1.262 & 1.998 \\
Data set II & 0.100 & 12.80 & 15.00 & 19.25 & 30.00 & 49.00 \\
Data set III & 0.047 & 0.395 & 0.841 & 1.341 & 2.178 & 4.033 \\
\hline
\end{tabular}

In the following, our proposed model is compared with some other competitive lifetime distributions, namely:

1. Power function distribution (PFD) [17]:

Its pdf is given in Definition 1.

2. Transmuted power function distribution (TPFD) [12]:

Its pdf is given by

$$
f_{T P F}(x ; \alpha, \beta, \lambda)=\frac{\alpha x^{\alpha-1}}{\beta^{\alpha}}\left(1+\lambda-2 \lambda\left(\frac{x}{\beta}\right)^{\alpha}\right), 0<x<\beta, \alpha>0,|\lambda| \leq 1 .
$$

3. Log-Normal distribution (LNorm) [23]:

Its pdf is given by

$$
f_{L N o r m}(x ; \mu, \sigma)=\frac{1}{x \sigma \sqrt{2 \pi}} \exp \left\{-\frac{(\ln x-\mu)^{2}}{2 \sigma^{2}}\right\}, \quad x \geq 0,-\infty<\mu<\infty, \quad \sigma>0 .
$$

4. Inverse Gaussian distribution (InvG) [24]:

Its pdf is given by

$$
f_{\mathrm{InvG}}(x ; \mu, \lambda)=\left(\frac{\lambda}{2 \pi x^{3}}\right)^{1 / 2} \exp \left\{\frac{-\lambda(x-\mu)^{2}}{2 \mu^{2} x}\right\}, \quad x \geq 0, \mu, \lambda>0 .
$$

These models are fitted to the above data sets. The MLEs, the Akaike information criterion (AIC), Bayesian information criterion (BIC), and the Kolmogorov-Smirnov statistic (K-S) for the above distributions as well as our proposed model are evaluated and the results are reported in Tables 4, 5, and 6 for data set I, II, and III, respectively. According to the values of AIC and BIC, our proposed model provides better fit than the competing distribution which confirm its superiority of the other distributions. According to the values of K$\mathrm{S}$ statistic, our proposed model provides better fit than the other models for data set I and data set II. For data set III, the TPFD provides better fit than the other models.

Moreover, to assess if the model is appropriate, Figures 3, 4, and 5 depict the histograms, the plots of the fitted PFD, TPFD, CTPFD, LNorm, and InvG density functions and their survival functions and the plots of the empirical distributions for data sets I, II and III, respectively. In addition, Q-Q plot and P-P plot are also depicted. It can be concluded that our new proposed distribution is a very suitable model for the above data sets. 
Table 4. The MLEs, -2LL, AIC, BIC, and $K$-S values for data set I under different models

\begin{tabular}{|c|c|c|c|c|c|c|c|}
\hline Distribution & \multicolumn{3}{|c|}{ Parameter estimates } & $-2 \mathrm{LL}$ & $\mathrm{AIC}$ & $\mathrm{BIC}$ & K-S \\
\hline $\operatorname{PFD}(\alpha, \beta)$ & 0.7254 & 1.9984 & - & 253.64 & 257.64 & 264.24 & 0.0605 \\
\hline $\operatorname{TPFD}(\alpha, \beta, \lambda)$ & 0.8170 & 1.9984 & 0.2615 & 251.75 & 257.75 & 267.65 & 0.0591 \\
\hline $\operatorname{CTPFD}(\alpha, \beta, \lambda)$ & 0.8934 & 1.9984 & 0.7107 & 250.06 & 256.06 & 261.96 & 0.0456 \\
\hline $\operatorname{LNorm}(\mu, \sigma)$ & 0.0000 & 1.3796 & - & 420.73 & 424.73 & 431.33 & 0.3078 \\
\hline $\operatorname{InvG}(\mu, \lambda)$ & 0.8054 & 0.2034 & - & 472.77 & 476.77 & 483.37 & 0.2901 \\
\hline
\end{tabular}

Table 5. The MLEs, -2LL, AIC, BIC, and $K$-S values for data set II under different models

\begin{tabular}{|c|c|c|c|c|c|c|c|}
\hline Distribution & \multicolumn{3}{|c|}{ Parameter estimates } & $-2 \mathrm{LL}$ & AIC & $\mathrm{BIC}$ & $\mathrm{K}-\mathrm{S}$ \\
\hline PFD $(\alpha, \beta)$ & 0.7242 & 49.0000 & - & 827.05 & 831.04 & 836.41 & 0.1786 \\
\hline $\mathrm{TPl}$ & 305 & & & 823.62 & 829.62 & 837.67 & 0.1775 \\
\hline $\mathrm{CTF}$ & 712 & 49.0000 & -0.673 & 7 & 827.97 & 836.02 & 0.1544 \\
\hline LNol & 2.4938 & 1.4111 & - & 919.50 & 923.50 & 928.86 & 0.2890 \\
\hline $\operatorname{InvG}(\mu, \lambda)$ & 19.2465 & 1.6159 & - & 1062.66 & 1066.66 & 1072.03 & 0.5559 \\
\hline
\end{tabular}

Table 6. The MLEs, -2LL, AIC, BIC, and $K$-S values for data set III under different models

\begin{tabular}{|c|c|c|c|c|c|c|c|}
\hline Distribution & \multicolumn{3}{|c|}{ Parameter estimates } & $-2 \mathrm{LL}$ & AIC & BIC & $\mathrm{K}-\mathrm{S}$ \\
\hline $\operatorname{PFD}(\alpha, \beta)$ & 0.3769 & 15.0800 & - & 203.46 & 207.46 & 211.28 & 0.1551 \\
\hline $\operatorname{TPFD}(\alpha, \beta, \lambda)$ & 0.4736 & 15.0800 & 0.5756 & 199.66 & 205.66 & 211.40 & 0.1349 \\
\hline $\operatorname{CTPFD}(\alpha, \beta, \lambda)$ & 0.5176 & 15.0911 & 1.0000 & 199.20 & 205.20 & 210.93 & 0.1378 \\
\hline $\operatorname{WRD}(\alpha, \beta, \lambda)$ & 0.0583 & 1.7925 & - & 206.06 & 210.06 & 213.89 & 0.1463 \\
\hline WBXII $(\alpha, \beta, a, b)$ & 3.3432 & 0.2946 & - & 211.69 & 215.69 & 219.52 & 0.2478 \\
\hline
\end{tabular}
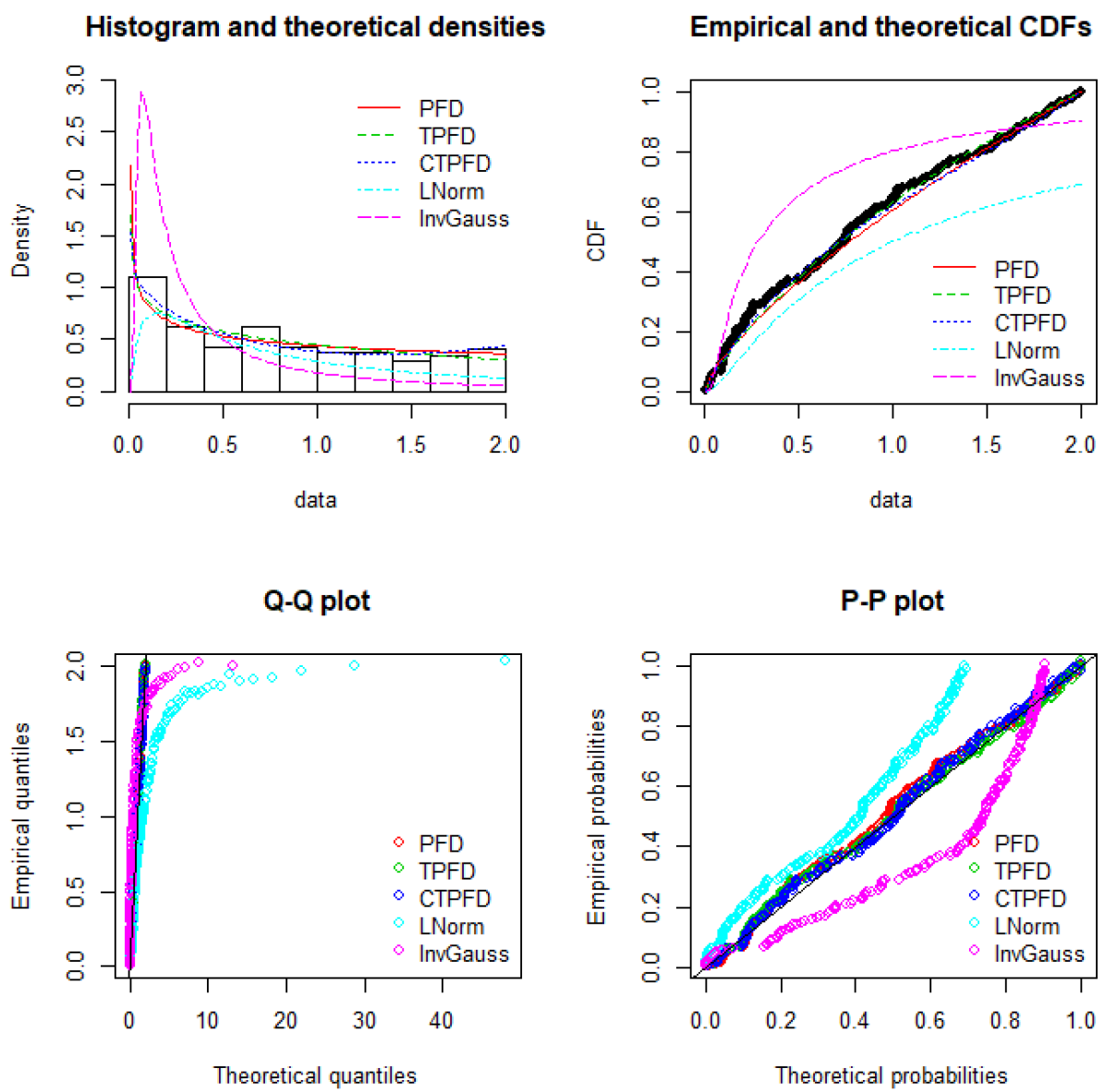

Figure 3. Histogram, density functions, survival function, $Q-Q$ plot and $P-P$ plot for data set I 
Histogram and theoretical densities

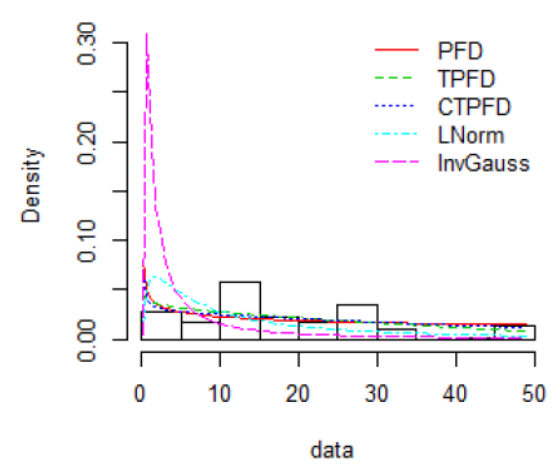

Q-Q plot

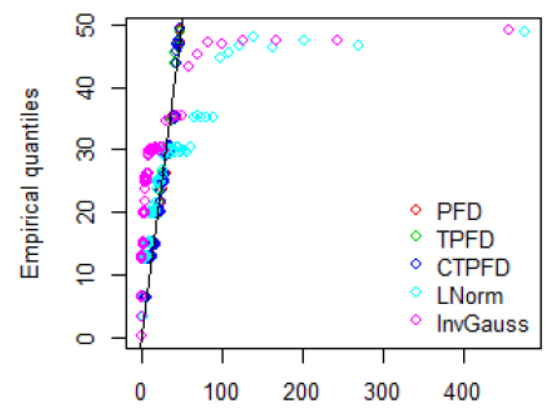

Theoretical quantiles

\section{Empirical and theoretical CDFs}

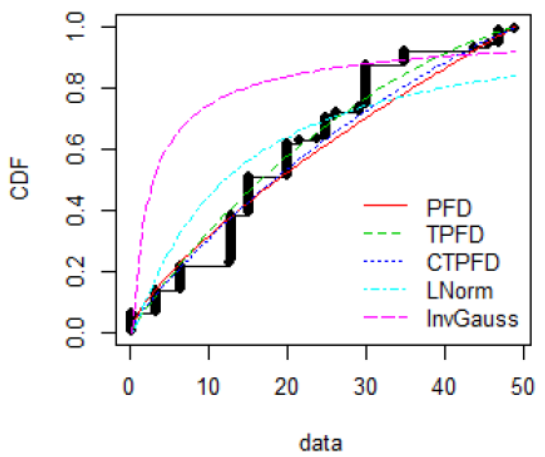

P.P plot

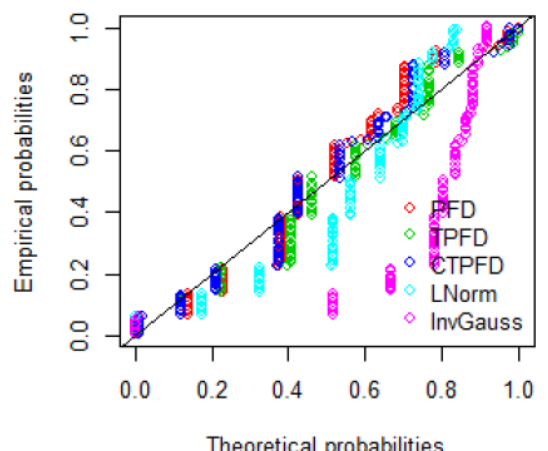

Figure 4. Histogram, density functions, survival function, $Q-Q$ plot and $P-P$ plot for data set II

Histogram and theoretical densities

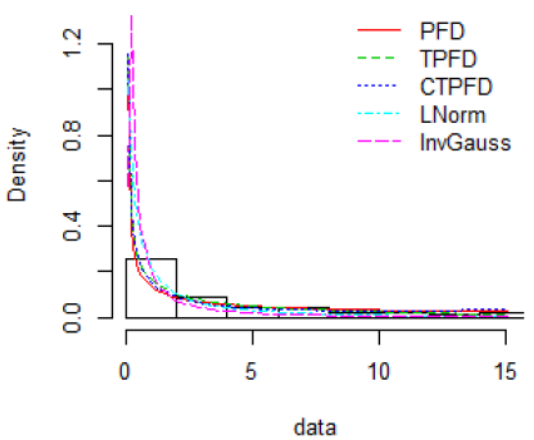

Q-Q plot

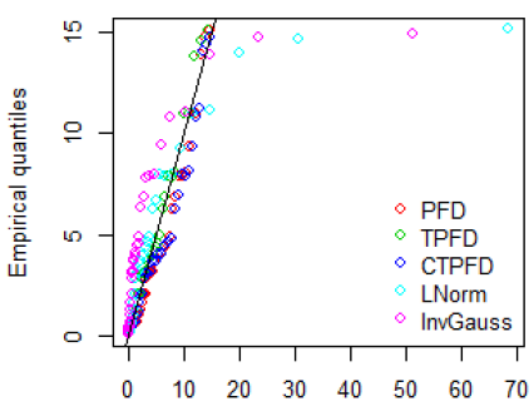

Theoretical quantiles

\section{Empirical and theoretical CDFs}

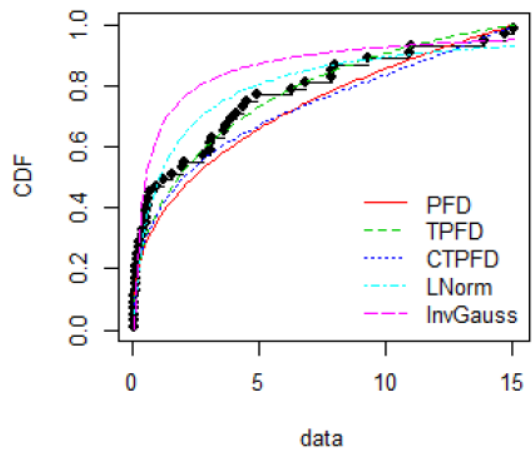

P.P plot

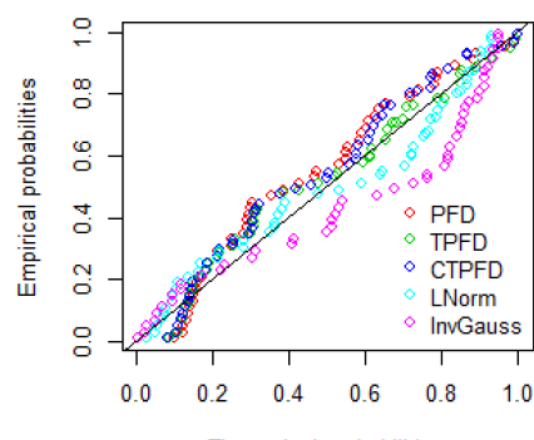

Theoretical probabilities

Figure 5. Histogram, density functions, survival function, $Q-Q$ plot and $P-P$ plot for data set III 


\section{CONCLUSION}

In this paper, the cubic transmuted power function distribution, which generalizes the power function and transmuted power function distributions, is investigated. Several statistical and mathematical relations of this distribution are obtained. Analytical forms of the pdf, cdf, reliability function, and cumulative hazard function are obtained. Density function of order statistic is also obtained. Explicit formulae for the moments, conditional moment, moment generating function, and some descriptive statistics are derived. Finally, moment matching estimation is used to estimate the distribution parameters. It is shown that the new proposed distribution equips better fits than some other distributions by using a real application. The new model may draw wider applications in reliability analysis.

\section{ACKNOWLEDGMENTS}

The authors wish to thank the associated editor and referees for their constructive comments which improve the final version of this paper. Moreover, the authors would like to acknowledge their universities (Tabuk University, Palestine Polytechnic University, Persian Gulf University) for giving them moral and technical support to carry out research work.

\section{CONFLICTS OF INTEREST}

No conflict of interest was declared by the authors.

\section{REFERENCES}

[1] Azzalini, A., "A class of distributions which includes the normal ones", Scandinavian Journal of Statistics, 12:171-178, (1985).

[2] Gupta, R., and Gupta, R., “Generalized skew-normal model”, Test, 13:501-524, (2004).

[3] Jamalizadeh, A., Behboodian, J., and Balakrishnan, N., "A two-parameter generalized skew-normal distribution", Statistical and Probability Letters, 78:1722-1728, (2008).

[4] Sharafi, M., and Behboodian, J., "The Balakrishnan skew-normal density”, Statistical Papers, 49:769778, (2008).

[5] Elal-Olivero, D., “Alpha-skew-normal distribution”, Proyecciones Journal of Mathematics, 29:224-240, (2010).

[6] Sharafi, M., Sajjadnia, Z., and Behboodian, J., "A new generalization of alpha-skew-normal distribution", Communications in Statistics - Theory and Methods, 46:6098-6111, (2017).

[7] Shaw, W., and Buckley, I., "The alchemy of probability distributions: beyond Gram-Charlier expansions, and a skew-kurtotic-normal distribution from a rank transmutation map", arXiv preprint arXiv:0901.0434, (2009).

[8] Aryal, G. R., and Tsokos, C. P., "On the transmuted extreme value distribution with application", Nonlinear Analysis: Theory, Methods \& Applications, 71(12):1401-1407, (2009).

[9] Elbatal, I., "Transmuted generalized inverted exponential distribution", Economic Quality Control, 28:125-133, (2013).

[10] Merovci, F., “Transmuted Rayleigh distribution”, Austrian Journal of Statistics, 42:21-31, (2013). 
[11] Oguntunde, P., and Adejumo, O., “The transmuted inverse exponential distribution”, International Journal of Advanced Statistics and Probability, 3:1-7, (2015).

[12] Haq, M., Butt, N., Usman, R., and Fattah, A., "Transmuted power function distribution”, Gazi University Journal of Science, 29:177-185, (2016).

[13] Shahzad, M. N., and Asghar, Z., "Transmuted power function distribution: A more flexible distribution", Journal of Statistics and Management Systems, 19(4):519-539, (2016).

[14] Granzotto, D. C. T., Louzada, F., and Balakrishnan, N., "Cubic rank transmuted distributions: inferential issues and applications", Journal of Statistical Computation and Simulation, 87(14):2760-2778, (2017).

[15] AL-Kadim, K. A., and Mohammed, M. H., "The cubic transmuted Weibull distribution", Journal of University of Babylon for Pure and Applied Sciences, 25(3):862-876, (2017).

[16] Rahman, M. M., Al-Zahrani, B., and Shahbaz, M. Q., "A general transmuted family of distributions", Pakistan Journal of Statistics and Operation Research, 14(2):451-469, (2018).

[17] Meniconi, M., and Barry, D. M., "The power function distribution: A useful and simple distribution to assess electrical component reliability”, Microelectronics Reliability, 36(9):1207-1212, (1996).

[18] Zaka, A., and Akhter, A. S., "Modified moment, maximum likelihood and percentile estimators for the parameters of the power function distribution", Pakistan Journal of Statistics and Operation Research, 10(4):369-388, (2014).

[19] Ahmad, A., and Akhter, A. S., "Kurtosis statistics with reference to power function distribution." Journal of Statistics, 23:123-140, (2016).

[20] R Core Team, "R: A Language and environment for statistical computing”, R Foundation for Statistical Computing, Vienna, Austria, (2018). URL https://www.R-project.org/.

[21] Delignette-Muller M. L., and Dutang C., "Fitdistrplus: An R package for fitting distributions", Journal of Statistical Software, 64(4):1-34, (2015).

[22] Murthy, D. P., Xie, M., and Jiang, R., "Weibull models (Vol. 505)”, John Wiley and Sons, (2004).

[23] Johnson, N. L., Kotz, S., and Balakrishnan, N., "14: Lognormal distributions", Continuous univariate distributions. Vol. 1, Wiley Series in Probability and Mathematical Statistics: Applied Probability and Statistics (2nd ed.), New York: John Wiley \& Sons (1994).

[24] Chhikara, R., and Folks, L., "The inverse Gaussian distribution: theory, methodology and applications", New York: Marcel Dekker, (1989). 